\title{
Étude de l'influence de la hauteur de piégeage sur les captures de pucerons ailés en différents sites et sur plusieurs années
}

\author{
M Hullé, É Vessier, Y Robert, J Rouzé-Jouan \\ INRA, Centre de recherches de Rennes, laboratoire de la chaire de Zoologie, BP 29, F35650 Le Rheu, France
}

(Reçu le 22 octobre 1992; accepté le 18 mai 1993)

\begin{abstract}
Résumé - La variabilité de l'influence de la hauteur de piégeage sur les captures de pucerons ailés par pièges jaunes a été étudiée en comparant les résultats obtenus dans 7 sites géographiquement distants et durant 5 années consécutives. Tous les pièges ont été disposés dans le même environnement cultural afin de minimiser les sources exogènes de variabilité. L'analyse a porté sur près de 40000 pucerons et 121 taxons et a permis de classer les 23 espèces les mieux capturées en 4 groupes : 6 espèces dont les captures sont régulièrement influencées par la hauteur de piégeage. Quel que soit le site ou l'année, 5 espèces dont les captures subissent moins régulièrement l'effet de la hauteur de piégeage, 6 espèces pour lesquelles l'effet dû à la hauteur de piégeage est faible ou instable, et enfin 6 espèces dont les captures semblent indépendantes de la hauteur de piégeage. Parmi les espèces montrant une différence significative de capturabilité, 8 sont piégées en plus grand nombre à $0,7 \mathrm{~m}$ et 3 à $0,0 \mathrm{~m}$. Ces résultats montrent cependant que le principal facteur d'hétérogénéité réside dans les fluctuations interannuelles qui permettent d'expliquer environ $50 \%$ de la variabilité observée dans les captures.
\end{abstract}

aphide / piégeage / piège jaune / densité aérienne

Summary - Study of the effect of the height of trapping on catches of alate aphids at different sites and over several years. The variability in the influence of the height of trapping on the abundance of catches of alate aphids was studied via comparisons between 2 heights of trapping at 7 different sites operating over $5 \mathrm{yr}$ in succession. All the traps were set in the same cultural environment in order to limit the influence of other possible factors of variability. The study was based on catches of nearly 40000 individuals and 121 taxa. It allowed classification of the 23 more abundant species into 4 groups: 6 species whose catches were regularly dependent on the height of trapping, 5 species whose catches were also related to the height of trapping but less regularly, 6 species for which the height effect was weak or irregular and 6 species showing no effect. Among the 11 first species, 8 were sampled at $0.7 \mathrm{~m}$ in a higher number than at $0.0 \mathrm{~m}$ and 3 were better sampled at $0.0 \mathrm{~m}$. The main factor of heterogeneity remained annual variations which constituted $50 \%$ of the total variability observed in the data set.

aphid / sampling / yellow water trap / aerial density

\section{INTRODUCTION}

L'estimation de la densité aérienne des populations de pucerons ailés par observation directe est difficile, voire impossible (Robert et al, 1988). II est donc, le plus souvent, nécessaire de recourir à des techniques de piégeage.

Certaines de ces techniques de piégeage permettent théoriquement une estimation du nombre de captures par volume d'air échantilloné et par unité de temps. C'est le cas des pièges à filtre (Taylor et Palmer, 1972) qui retiennent les insectes malgré eux. L'importance des captures ne dépend donc a priori que des conditions d'exposition des pièges (hauteur, environnement végétal, direction et vitesse du vent...) et de la densité aérienne des populations au moment de l'échantillonnage. Les pièges à succion (Johnson et Taylor, 1955) ap- 
partiennent à cette catégorie. De tels pièges sont particulièrement adaptés pour étudier les mouvements des populations aériennes.

Les autres techniques de piégeage sont fondées généralement sur l'utilisation d'une source colorée ou lumineuse exerçant une attraction sur les pucerons ailés. C'est le cas des pièges à impact ou des pièges lumineux (Taylor et Palmer, 1972). On ne connaît pas le volume d'attraction de ces pièges ; ils ne permettent donc théoriquement que des comparaisons dans le temps des niveaux de populations. Cependant et outre les conditions d'exposition des pièges et la densité aérienne des populations échantillonnées, les captures réalisées par ces pièges dépendent également du comportement spécifique et individuel des pucerons, de leur capacité à percevoir la source colorée et à se diriger vers elle. Les pièges à eau colorés, décrits par Moericke (1951), appartiennent à cette catégorie. De tels pièges sont très souvent utilisés dans la pratique des avertissements agricoles pour détecter la contamination d'une culture par la fraction ailée de la population (Robert et al, 1974).

Parmi les conditions d'exposition des pièges, la hauteur à laquelle ils sont installés peut influencer aussi bien la densité d'une espèce particulière que la composition spécifique du peuplement capturé. Plusieurs auteurs ont étudié les captures de pucerons en relation avec la hauteur de piégeage. Taylor (1974) a décrit pour des homoptères, dont principalement des pucerons, mais aussi pour d'autres insectes, la relation liant l'intensité des captures (y) à la hauteur de piégeage (z). Cette relation, établie avec des pièges à succion, est de type :

$$
\log (y)=b \times \log (z)
$$

avec $b$ négatif.

L'intensité des captures décroît donc avec la hauteur de piégeage, mais seulement au-dessus d'une couche limite de vol (boundary layer). $\dot{A}$ l'intérieur de cette couche limite, généralement située entre $0,2 \mathrm{~m}$ et $1,5 \mathrm{~m}$, la densité des captures est stable, voire croissante, avec la hauteur. Taylor utilise ce concept de boundary layer pour décrire la couche d'air près du sol à l'intérieur de laquelle les insectes sont supposés contrôler leurs propres mouvements grâce à une vitesse de vol supérieure à la vitesse du vent. Au-dessus, ils sont supposés être entraînés par les courants aériens.

Les études concernant l'influence de la hauteur de piégeage sur les captures ont été le plus souvent restreintes à un seul site et/ou à une période limitée dans le temps (A'Brook, 1973 ; Robert et al, 1974 ; Taylor, 1974 ; Labonne et al, 1983 ; Labonne et al, 1989 ; Hullé et al, 1992). La stabilité spatio-temporelle des relations ainsi décrites est donc incertaine.

Dans cet article, nous comparons les captures de pucerons par pièges jaunes à eau à 2 hauteurs différentes dans le but de vérifier la stabilité de l'influence de ce facteur sur la composition des captures. Le dispositif expérimental a pour cela été répété sur plusieurs années et dans différents sites géographiquement distants. Dans chacun des sites, les pièges ont été placés dans le même environnement végétal immédiat, de façon à atténuer le plus possible l'influence de ce facteur. Les 2 hauteurs testées, situées a priori à l'intérieur de la couche limite décrite par Taylor, correspondent à celles habituellement utilisées dans les opérations de surveillance par pièges jaunes des populations de pucerons ravageurs des cultures.

\section{MATÉRIEL ET MÉTHODES}

Nos observations ont été extraites d'un dispositif expérimental mis en place en Bretagne (France) dans le cadre d'une étude sur l'épidémiologie des viroses de la pomme de terre transmises par pucerons (Robert et al, 1974). De ce dispositif nous n'avons retenu que certains sites et certaines années afin de disposer d'un ensemble de données suffisamment homogène du point de vue des conditions climatiques et des caractéristiques des pièges.

\section{Sites géographiques}

Sept sites ont été retenus, tous situés en zone de production de plants de pomme de terre (fig 1).

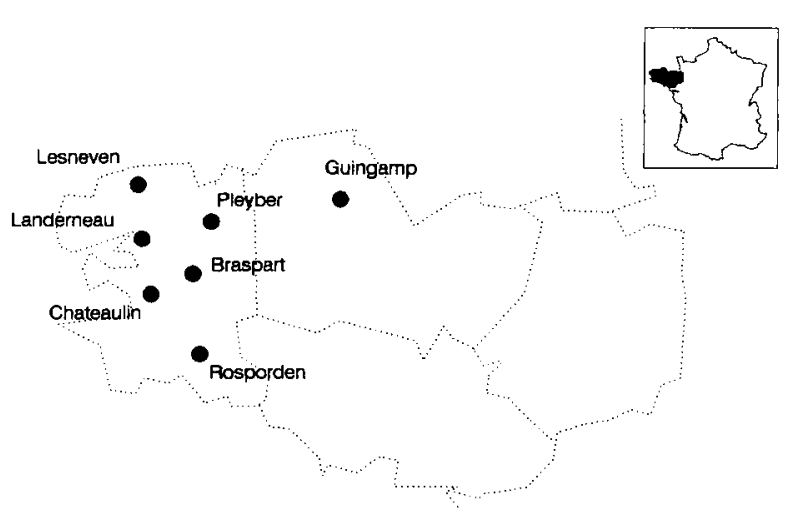

Fig 1. Répartition des lieux de captures des pucerons. 


\section{Périodes}

Les observations ont été poursuivies pendant 5 années, de 1974 à 1978, dans les différents sites. Les pièges ont été mis en place entre le début du mois de mai et la fin du mois de juillet, c'est-à-dire pendant les vols de contamination et de dissémination (Robert et Rouzé-Jouan, 1976).

\section{Pièges}

En chacun des sites, 4 pièges ont été mis en place : 2 pièges à $0,0 \mathrm{~m}$ et 2 pièges à $0,7 \mathrm{~m}$. Nous disposons ainsi de 35 comparaisons possibles entre les 2 hauteurs ( 5 années $\times 7$ sites) et de 2 répétitions pour chacune.

Les pièges sont des cuvettes carrées de 0,3 m de côté. Elles ont été disposées aux 4 coins d'un carré de $10 \mathrm{~m}$ de côté, au milieu de parcelles servant à la vérification a posteriori de la qualité sanitaire des plants de pomme de terre.

Les cuvettes sont peintes en jaune avec un domaine spectral de diffusion compris entre 5000 et $6300 \AA$, et un maximum de diffusion pour une longueur d'onde de $5800 \AA$. Les cuvettes ont $0,12 \mathrm{~m}$ de profondeur et sont remplies aux deux tiers d'eau additionnée de teepol.

\section{Récoltes et déterminations}

Les pucerons sont récoltés 2 ou 3 fois par semaine. Ils sont conservés dans l'alcool à $70 \%$ et déterminés au microscope stéréoscopique.

\section{Analyse des données}

Selon le type de résultats, plusieurs méthodes descriptives et statistiques ont été utilisées :

- analyse de variance à 3 facteurs : site, année et hauteur de piégeage ; lorsque cela a été nécessaire, ces analyses ont été complétées par un test de comparaison de moyennes suffisamment conservatif, le test de Newman-Keuls (logiciel Statitcf, Tranchefort et al, 1987) ;

- analyse factorielle des correspondances (AFC) pour les tableaux d'effectifs croisant les espèces et les 70 cas de piégeage (5 années $\times 7$ sites $\times 2$ hauteurs) (logiciel Spad, Lebart et al, 1988) ;

- test des signes pour comparer le nombre d'occasions où les captures d'une hauteur donnée sont supérieures ou inférieures aux captures de l'autre hauteur (Lebart et al, 1979).

\section{RÉSULTATS}

\section{Caractéristique globable des captures : effectif total et nombre d'espèces}

L'analyse des effectifs totaux moyens, calculés sur les 2 répétitions pour chaque site, année et hauteur de piégeage (tableau I) révèle que, dans 24 cas sur 35 , les captures sont plus élevées à $0,7 \mathrm{~m}$ (243 individus par an en moyenne) qu'à 0,0 m (204 individus par an en moyenne).

Ce phénomène est régulier pour 3 sites : Lesneven, Guinguamp et Rosporden. II est observé tous les ans sauf en 1977 à Braspart. II est observé de 1974 à 1976 à Châteaulin et en 1975, 1977 et 1978 à Pleyber. Les pièges de Landerneau ont un comportement inverse, en capturant régulièrement plus de pucerons à $0,0 \mathrm{~m}$ qu'à $0,7 \mathrm{~m}$.

L'analyse de la variance de ces données brutes après transformation logarithmique (tableau II) indique qu'il existe bien un effet dû à la hauteur de piégeage. Cet effet est faible par rapport aux variations dues au site et surtout celles dues à l'année. Le site géographique interagit fortement avec la hauteur de piégeage. Ainsi, l'inversion d'effet observée à Landerneau est significative. À Pleyber, les 2 hauteurs ne sont pas différenciées par leur niveau moyen de captures. L'interaction année/hauteur est due pour l'essentiel à une absence d'effet hauteur en 1974 et en 1977.

La différence d'efficacité globale due à la hauteur de piégeage peut être très importante, de l'ordre de 1 à 5 fois plus de captures à $0,7 \mathrm{~m}$ qu'à $0,0 \mathrm{~m}$. Aucune relation n'a été décelée entre l'importance de cette différence et le niveau de capture. Les rapports les plus élevés ont été observés à Rosporden.

De même que pour l'effectif total, le nombre d'espèces est également plus élevé en moyenne dans les pièges à $0,7 \mathrm{~m}$ (27 espèces) que dans les pièges à $0,0 \mathrm{~m}$ (23 espèces). II existe également une interaction entre la hauteur et le site, mais plus entre la hauteur et l'année (tableau III).

L'interaction site-année-hauteur est due essentiellement à Landerneau, dont les résultats sont inverses à la moyenne des autres pièges. Tous les autres sites capturent, en effet, plus d'espèces à $0,7 \mathrm{~m}$ qu'à $0,0 \mathrm{~m}$, à l'exception de 
Tableau I. Effectif annuel moyen des pucerons capturés dans chaque site, hauteur et année.

\begin{tabular}{|c|c|c|c|c|c|c|}
\hline & 1974 & 1975 & 1976 & 1977 & 1978 & Moyenne \\
\hline \multicolumn{7}{|l|}{ Pleyber } \\
\hline $0,00 \mathrm{~m}$ & 384 & 318 & 790 & 252 & 30 & 355 \\
\hline $0,70 \mathrm{~m}$ & 332 & 320 & 643 & 255 & 36 & 317 \\
\hline \multicolumn{7}{|l|}{ Lesneven } \\
\hline $0,00 \mathrm{~m}$ & 77 & 136 & 206 & 90 & 19 & 106 \\
\hline 0,70 & 101 & 264 & 505 & 107 & 33 & 202 \\
\hline \multicolumn{7}{|l|}{ Landerneau } \\
\hline $0,00 \mathrm{~m}$ & 404 & 432 & 1067 & 80 & 56 & 408 \\
\hline $0,70 \mathrm{~m}$ & 224 & 255 & 338 & 46 & 44 & 181 \\
\hline \multicolumn{7}{|l|}{ Guingamp } \\
\hline $0,00 \mathrm{~m}$ & 260 & 119 & 202 & 7 & 47 & 127 \\
\hline $0,70 \mathrm{~m}$ & 256 & 254 & 293 & 9 & 86 & 180 \\
\hline \multicolumn{7}{|l|}{ Rosporden } \\
\hline $0,00 \mathrm{~m}$ & 201 & 443 & 254 & 21 & 32 & 190 \\
\hline $0,70 \mathrm{~m}$ & 356 & 724 & 823 & 105 & 141 & 430 \\
\hline \multicolumn{7}{|l|}{ Châteaulin } \\
\hline $0,00 \mathrm{~m}$ & 103 & 195 & 247 & 204 & 134 & 177 \\
\hline $0,70 \mathrm{~m}$ & 140 & 495 & 641 & 122 & 66 & 293 \\
\hline \multirow{2}{*}{\multicolumn{7}{|c|}{ Braspart }} \\
\hline $0,00 \mathrm{~m}$ & 66 & 93 & 84 & 69 & & 68 \\
\hline $0,70 \mathrm{~m}$ & 102 & 146 & 139 & 55 & 58 & 100 \\
\hline \multicolumn{7}{|l|}{ Moyenne } \\
\hline $0,00 \mathrm{~m}$ & 214 & 248 & 407 & 103 & 49 & 204 \\
\hline $0,70 \mathrm{~m}$ & 216 & 351 & 483 & 100 & 66 & 243 \\
\hline
\end{tabular}

quelques cas ponctuels (Pleyber en 1974, Châteaulin en 1978 et Braspart en 1977).

Ces résultats globaux montrent une efficacité des pièges généralement meilleure à $0,7 \mathrm{~m}$ qu'à $0,0 \mathrm{~m}$, tant en nombre d'espèces capturées qu'en nombre total d'individus piégés. La hauteur de $0,7 \mathrm{~m}$ semble donc préférable pour une étude globale des peuplements de pucerons. Mais ce rapport d'efficacité globale peut varier, voire s'inverser, pour certains sites ou certaines années. II est nécessaire de vérifier si ces inversions sont dues à plusieurs espèces majeures, ce qui pourrait remettre en cause l'hypothèse d'homogénéité du protocole d'échantillonnage, ou bien à un très petit nombre d'espèces seulement, ce qui pourrait alors révéler un effet local dû à l'environnement immédiat.

Par ailleurs, il est important de décrire également ces rapports d'efficacité espèce par espèce, car ils peuvent varier en fonction de la biologie et du comportement des différentes espèces.

\section{Comparaison des captures par espèce}

\section{Choix des espèces}

Au total, 121 taxons ont été capturés sur l'ensemble des 7 sites de 1974 à 1978 .

Soixante-dix pour cent des espèces capturées sont très peu fréquentes. Parmi ces espèces rarement piégées, certaines ne l'ont été qu'à $0,7 \mathrm{~m}$. II s'agit de Sipha glyceriae Kaltenbach, Megourella purpurea Hille, Lipaphis erysimi Kaltenbach, Betulaphis quadrituberculata Kaltenbach, Brachycaudus sedi Jacob, Aphis idaei Van der Goot, Thripsaphis cyperi Walker, Schizaphis graminum Rondani, Ceruraphis eriophori Walker et Eulachnus agilis Kaltenbach. D'autres espèces, à l'inverse, n'ont été capturées qu'à 0,0 m. Ce sont Diuraphis frequens Walker, Cryptomyzus korschelti Börner, Atheroides serrulatus Haliday et Longicaudus trirhodus Walker. Mais ces espèces ne se prêtent pas à une 
Tableau II. Analyse de la variance du tableau des effectifs annuels, transformés en Log 10. Les tests de comparaison de moyennes concernent les 2 interactions simples faisant intervenir la hauteur de piégeage.

\begin{tabular}{|c|c|c|c|c|c|c|c|}
\hline & $S C E$ & $D D L$ & Carrés moyens & Test $F$ & Prob & $E T$ & CV \\
\hline Var totale & 30,24 & 139 & 0,22 & & & & \\
\hline Site & 4,20 & 6 & 0,70 & 113,42 & 0,000 & & \\
\hline Année & 15,60 & 4 & 3,90 & 631,89 & 0,000 & & \\
\hline Hauteur & 0,51 & 1 & 0,51 & 81,96 & 0,000 & & \\
\hline Site-année & 6,99 & 24 & 0,29 & 47,17 & 0,000 & & \\
\hline Site-hauteur & 1,53 & 6 & 0,25 & 41,20 & 0,000 & & \\
\hline Année-hauteur & 0,11 & 4 & 0,03 & 4,45 & 0,003 & & \\
\hline Site-année-hauteur & 0,88 & 24 & 0,04 & 5,97 & 0,000 & & \\
\hline Var résiduelle & 0,43 & 70 & 0,01 & & & 0,08 & $3,7 \%$ \\
\hline
\end{tabular}

Interaction site-hauteur (test de Newman-Keuls, seuil 5\%)

Libellés Moyennes Groupe homogènes

$\begin{array}{lll}\text { Rosporden } & 0,70 \mathrm{~m} & 2,50 \\ \text { Landerneau } & 0,00 \mathrm{~m} & 2,38 \\ \text { Pleyber } & 0,00 \mathrm{~m} & 2,37 \\ \text { Pleyber } & 0,70 \mathrm{~m} & 2,36 \\ \text { Châteaulin } & 0,70 \mathrm{~m} & 2,31 \\ \text { Châteaulin } & 0,00 \mathrm{~m} & 2,22 \\ \text { Lesneven } & 0,70 \mathrm{~m} & 2,14 \\ \text { Landerneau } & 0,70 \mathrm{~m} & 2,11 \\ \text { Guingamp } & 0,70 \mathrm{~m} & 2,04 \\ \text { Rosporden } & 0,00 \mathrm{~m} & 2,03 \\ \text { Braspart } & 0,70 \mathrm{~m} & 1,96 \\ \text { Lesneven } & 0,00 \mathrm{~m} & 1,91 \\ \text { Guincamp } & 0,00 \mathrm{~m} & 1,86 \\ \text { Braspart } & 0,00 \mathrm{~m} & 1,79\end{array}$

A

2,38 B

$\begin{array}{ll}2,37 & B\end{array}$

B

Interaction année-hauteur (test de Newman-Keuls, seuil 5\%)

Libellès Moyennes Groupes homogènes

\begin{tabular}{|c|c|c|c|c|c|c|c|c|c|}
\hline 1976 & $0,70 \mathrm{~m}$ & 2,62 & $A$ & & & & & & \\
\hline 1975 & $0,70 \mathrm{~m}$ & 2,49 & & B & & & & & \\
\hline 1976 & $0,00 \mathrm{~m}$ & 2,46 & & B & & & & & \\
\hline 1975 & $0,00 \mathrm{~m}$ & 2,32 & & & C & & & & \\
\hline 1974 & $0,70 \mathrm{~m}$ & 2,28 & & & C & $\mathrm{D}$ & & & \\
\hline 1974 & $0,00 \mathrm{~m}$ & 2,23 & & & & $\mathrm{D}$ & & & \\
\hline 1977 & $0,70 \mathrm{~m}$ & 1,85 & & & & & $E$ & & \\
\hline 1977 & $0,00 \mathrm{~m}$ & 1,80 & & & & & $E$ & $F$ & \\
\hline 1978 & $0,70 \mathrm{~m}$ & 1,77 & & & & & & $\mathrm{~F}$ & \\
\hline 1978 & $0,00 \mathrm{~m}$ & 1,60 & & & & & & & $\mathrm{G}$ \\
\hline
\end{tabular}

analyse statistique, leurs effectifs étant trop faibles.

Nous avons retenu 23 espèces pour la suite de l'analyse. Ces espèces représentent en général plus de $80 \%$ des captures par hauteur de piégeage et par site.

\section{Analyse de la composition spécifique des captures}

Dans les tableaux IV et $\mathrm{V}$ sont fournis les pourcentages que chacune des 23 espèces retenues représente au sein des captures. Dans le tableau IV, les résultats sont présentés en fonc- 
Tableau III. Analyse de la variance du tableau des richesses spécifiques annuelles, transformées en Log 10 . Seules les interactions significatives et faisant intervenir la hauteur de piégeage sont détaillées.

\begin{tabular}{lrrrrrrl}
\hline & SCE & DDL & Carrés moyens & Test F & Proba & ET & CV \\
\hline Var totale & 6,32 & 139 & 0,05 & & & \\
Site & 0,36 & 6 & 0,06 & 20,55 & 0,0000 & & \\
Année & 3,77 & 4 & 0,94 & 322,74 & 0,0000 & & \\
Hauteur & 0,20 & 1 & 0,20 & 68,78 & 0,0000 & & \\
Site-année & 1,38 & 24 & 0,06 & 19,61 & 0,0000 & & \\
Site-hauteur & 0,21 & 6 & 0,03 & 11,82 & 0,0000 & & \\
Année-hauteur & 0,01 & 4 & 0,00 & 0,85 & 0,4986 & & \\
Site-année-hauteur & 0,19 & 24 & 0,01 & 2,66 & 0,0008 & 0,05 & $4,0 \%$ \\
Var résiduelle & 0,20 & 70 & 0,00 & & & &
\end{tabular}

Interaction site-hauteur

$\begin{array}{cccccccc} & \text { Pleyber } & \text { Rosporden } & \text { Lesneven } & \text { Châteaulin } & \text { Landerneau } & \text { Braspart } & \text { Guingamp } \\ & & & & & & & \\ 0,00 \mathrm{~m} & 1,35 & 1,32 & 1,29 & 1,38 & 1,41 & 1,26 & 1,22 \\ 0,70 \mathrm{~m} & 1,44 & 1,51 & 1,36 & 1,44 & 1,33 & 1,32 & 1,35\end{array}$

Interaction site-année-hauteur

1974

Valeurs moyennes sur l'ensemble des sites

$0,70 \mathrm{~m}$

1,45

1,50

1,54

1,52
1975

1,45

1,53

1,54

1,44
1976

1,47

1,53

1,56

1,48
1977

1978

1,09

1,17

1,13

1,23

1,15

1,25

1,02 tion de la hauteur de piégeage et de l'année. Dans le tableau $V$, les résultats sont présentés en fonction de la hauteur de piégeage et de la situation géographique. Le tableau complet, qui combine les 3 facteurs hauteur de piégeage, année et site, a été étudié par AFC (fig 2).

L'effet dû à la hauteur de piégeage sur la composition spécifique des captures est de même type pour l'ensemble des années et des sites et se caractérise par un changement comparable de coordonnées sur les deux premiers axes de I'AFC : diminution le long de l'axe 2 et augmentation le long de l'axe 1 lorsqu'on passe de $0,0 \mathrm{~m}$ à $0,7 \mathrm{~m}$. Ce changement de coordonnées est dû à une proportion plus importante de pucerons des arbres, Tuberculatus annulatus Hartig et Myzocallis castanicola Baker, d'un puceron des céréales, Metopolophium dirhodum Walker et de
Brevicoryne brassicae Linnaeus à $0,7 \mathrm{~m}$. II est dû également à des captures proportionnellement plus importantes d'Hyperomyzus lactucae Linnaeus à $0,0 \mathrm{~m}$.

L'effet dû à la hauteur de piégeage est plus ou moins sensible selon les sites ou les années. Mais la variabilité des captures due aux sites est moins importante que la variabilité due aux années. Les projections des points moyens de chacun des sites sont, en effet, regroupées au centre du premier plan de l'AFC (fig 2a) alors que les projections des points moyens de chaque année se répartissent sur l'ensemble du plan (fig 2b).

L'effet hauteur est particulièrement visible à Rosporden et à Lesneven. Landerneau fait exception par absence de déplacement le long de l'axe 1. Cela est dû surtout à l'année 1976 avec 


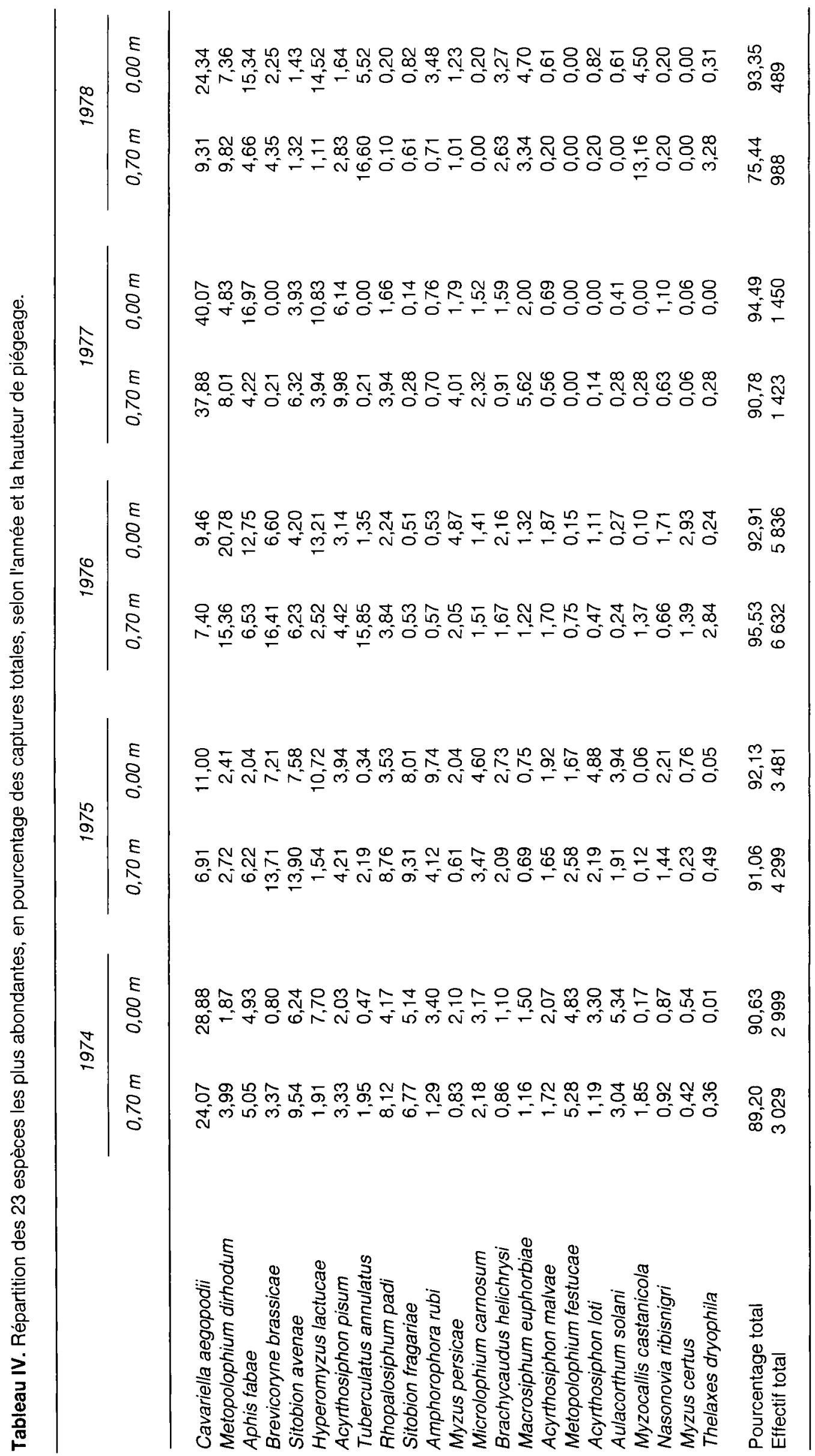




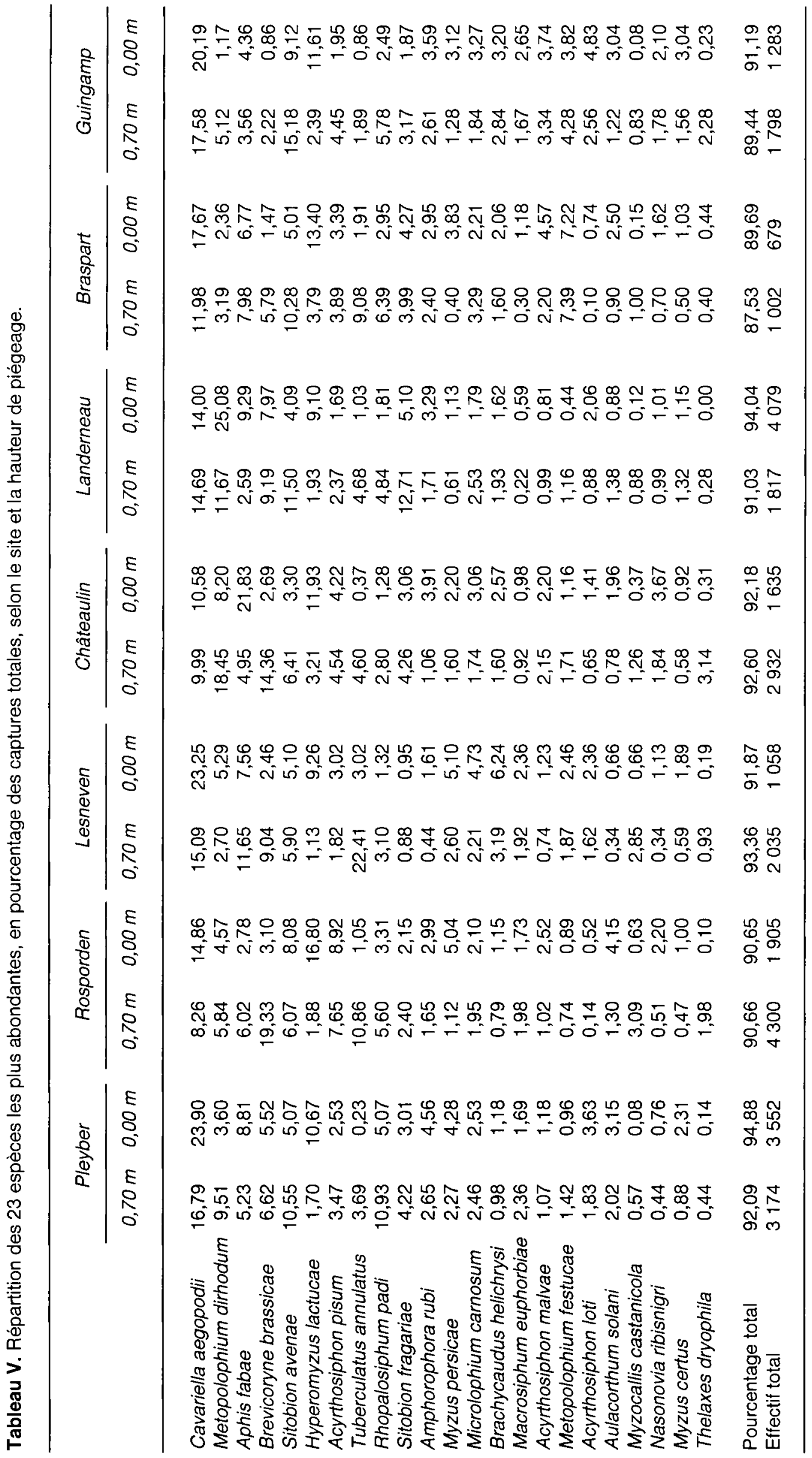


a

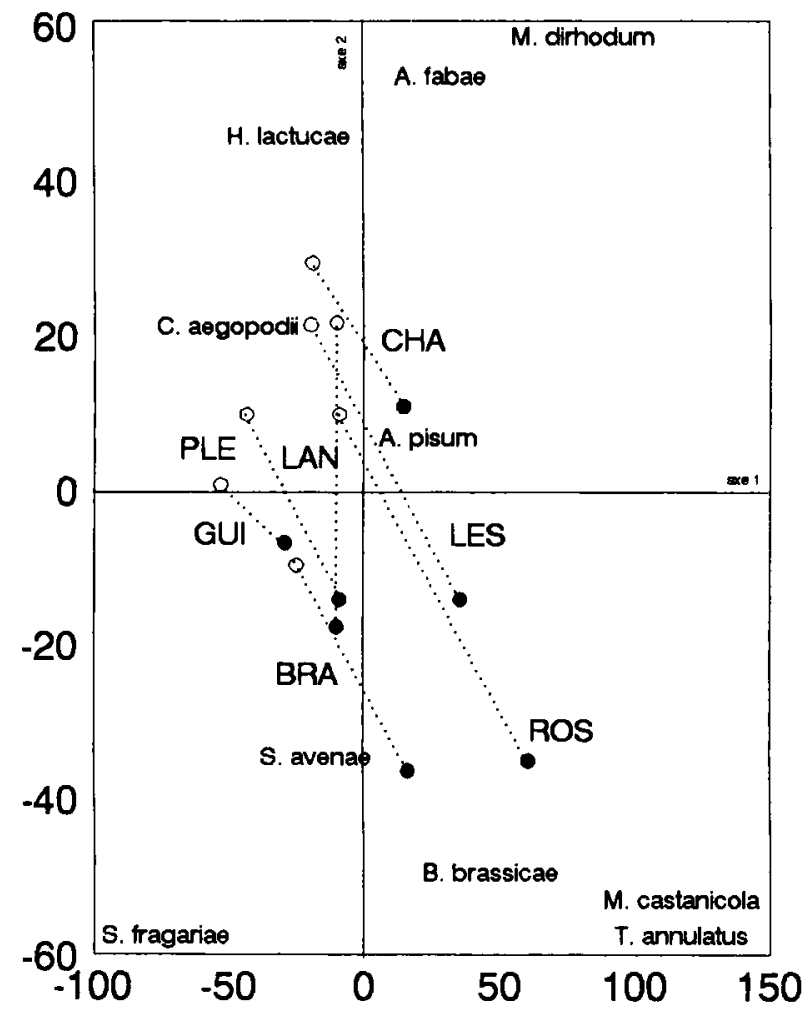

b

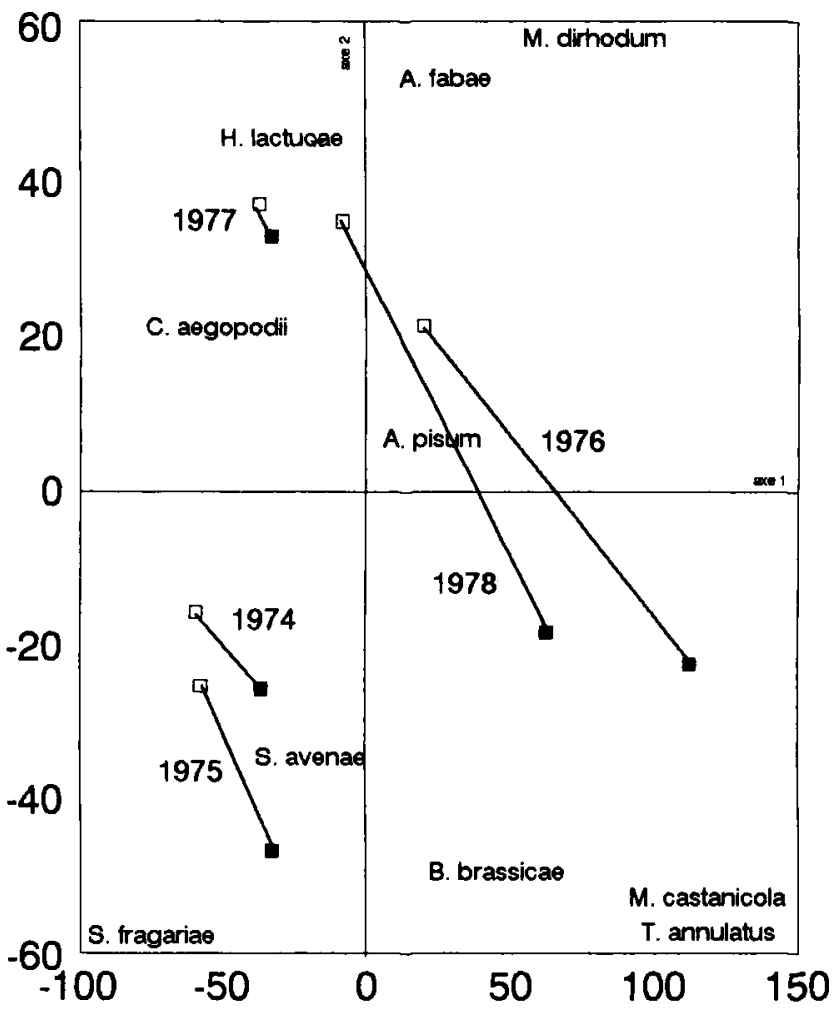

Fig 2. Reprèsentation graphique dans le plan des axes 1 et 2 (55\% d'inertie) de l'analyse factorielle des correspondances des effectifs annuels de pucerons dans les 70 occasions de piégeage ( 7 sites, 5 années, 2 hauteurs). a. Projection des points moyens site/ hauteur. b. Projection des points moyens année/hauteur (marques noires : piégeage à $0,7 \mathrm{~m}$; marques blanches : piégeage à $0,0 \mathrm{~m})$.

une proportion plus grande de $M$ dirhodum dans les captures réalisées à $0,0 \mathrm{~m}$. Les pièges de Guingamp sont très homogènes et les différences principales entre les captures des 2 hauteurs sont imputables essentiellement à Sitobion avenae Fabricius et $H$ lactucae.

L'effet dû à la hauteur de piégeage est peu visible en 1977, car il est masqué par une très forte proportion de Cavariella aegopodii Scopoli aux 2 hauteurs et, dans une moindre mesure, d'Acyrthosiphon pisum Harris. De même en 1974 et 1975 , les captures de $S$ avenae et Sitobion fragariae Walker sont proportionnellement importantes et confèrent à ces 2 années une spécificité décrite par leur projection sur le premier plan factoriel. L'effet dû à la hauteur de piégeage est particulièrement visible en 1976 et en 1978. Il est par ailleurs caractéristique de l'effet moyen avec des captures de pucerons des arbres et des céréales proportionnellement plus importantes à $0,7 \mathrm{~m}$ qu'à $0,0 \mathrm{~m}$, et des captures d'Aphis fabae Scopoli et d'H lactucae proportionnellement plus importantes à $0,0 \mathrm{~m}$ qu'à $0,7 \mathrm{~m}$.
La composition spécifique des captures est donc caractérisée par de nombreuses interactions entre la hauteur de piégeage, le site et l'année. Autrement dit, il n'existe pas de capture type, mais plutôt une distribution variable des effectifs au sein d'un groupe d'espèces dominantes qui représentent toujours environ $60 \%$ des captures totales.

\section{Analyse de l'efficacité des 2 hauteurs de piégeage sur les captures des différentes espèces}

L'effet dû à la hauteur de piégeage sur les captures des 23 espèces retenues peut être apprécié de différentes manières.

La première consiste à comparer les captures totales en cuvettes hautes aux captures totales en cuvettes basses. Ce rapport, assimilable à une efficacité, a été exprimé sous forme du pourcentage d'effectif selon la hauteur (tableau VI).

La deuxième est d'estimer la capturabilité relative, définie comme étant la probabilité pour 
Tableau VI. Effet de la hauteur de piégeage sur les captures des 23 espèces les plus abondantes.

\begin{tabular}{|c|c|c|c|c|c|c|}
\hline & \multicolumn{2}{|c|}{ Efficacité } & \multicolumn{2}{|c|}{ Capturabilité } & & \multirow[t]{2}{*}{ Type d'effet } \\
\hline & $0,70 \mathrm{~m}$ & $0,00 \mathrm{~m}$ & $0,70 \mathrm{~m}$ & $0,00 \mathrm{~m}$ & & \\
\hline Thelaxes dryophila & 93 & 7 & 96 & 4 & $\left({ }^{\star}\right)$ & 3 \\
\hline Myzocallis castanicola & 92 & 8 & 100 & 0 & ()$^{\star}$ & 3 \\
\hline Tuberculatus annulatus & 92 & 8 & 92 & 8 & $\left({ }^{\star}\right)$ & 3 \\
\hline Brevicoryne brassicae & 74 & 26 & 72 & 28 & $\left({ }^{*}\right)$ & 2 \\
\hline Rhopalosiphum padi & 71 & 26 & 83 & 17 & ()$\left.^{\star}\right)$ & 3 \\
\hline Sitobion avenae & 66 & 34 & 87 & 13 & ()$\left.^{*}\right)$ & 3 \\
\hline Acyrthosiphon pisum & 62 & 38 & 69 & 31 & $\left(^{*}\right)$ & 1 \\
\hline Sitobion fragariae & 60 & 40 & 67 & 33 & $\left({ }^{\star}\right)$ & 2 \\
\hline Metopolophium festucae & 58 & 42 & 65 & 35 & & 1 \\
\hline Macrosiphum euphorbiae & 57 & 43 & 58 & 42 & & 0 \\
\hline Metopolophium dirhodum & 50 & 50 & 80 & 20 & $(*)$ & 2 \\
\hline Microlophium carnosum & 50 & 50 & 58 & 42 & & 0 \\
\hline Acyrthosiphon malvae & 49 & 51 & 50 & 50 & & 0 \\
\hline Brachycaudus helichrysi & 49 & 51 & 53 & 47 & & 0 \\
\hline Cavariella aegopodii & 47 & 53 & 57 & 43 & & 0 \\
\hline Aphis fabae & 44 & 56 & 52 & 48 & & 0 \\
\hline Nasonovia ribisnigri & 41 & 59 & 32 & 68 & & 1 \\
\hline Myzus certus & 40 & 60 & 40 & 60 & & 1 \\
\hline Aulacorthum solani & 39 & 61 & 24 & 76 & $\left({ }^{\star}\right)$ & 2 \\
\hline Amphorophora rubi & 37 & 63 & 34 & 66 & & 1 \\
\hline Myzus persicae & 36 & 64 & 37 & 63 & & 1 \\
\hline Acyrthosiphon loti & 35 & 65 & 31 & 69 & $\left({ }^{\star}\right)$ & 2 \\
\hline Hyperomyzus lactucae & 19 & 81 & 3 & 97 & $\left({ }^{*}\right)$ & 3 \\
\hline
\end{tabular}

Efficacité : répartition des effectifs entre les 2 hauteurs exprimée en pourcentage ; capturabilité : pourcentage d'occasions où les captures ont été supérieures à une hauteur donnée ( ${ }^{*}$ : test des signes significatif à $5 \%$ ); type d'effet : $3:$ effet hauteur stable et important, 2 : effet hauteur presque stable, 1 : effet hauteur faible ou instable, $0:$ pas d'effet dû à la hauteur de piégeage.

une espèce donnée d'être capturée en plus grand nombre soit en cuvettes hautes, soit en cuvettes basses, indépendamment de l'effectif lui-même. Cette estimation est obtenue en rapportant le nombre de fois où une espèce est mieux capturée en cuvettes hautes, par exemple, au nombre total d'occasion de piégeage ( 5 années $\times 7$ sites). Le nombre de différences positives est alors testé par le test des signes. Les différences nulles entre cuvettes hautes et basses, qui correspondent surtout aux années où il n'y a aucune capture, ne sont pas prises en compte dans l'estimation d'une différence de capturabilité. Les résultats sont exprimés en pourcentage du nombre d'occasions et accompagnés d'un astérisque lorsque le test des signes est significatif (tableau VI).

La troisième manière, enfin, relève de l'analyse de la variance et consiste à apprécier les interactions entre l'éventuel effet dû à la hauteur de piégeage et les 2 autres facteurs de variabilité que sont l'année et le site. Ces analyses n'ont été effectuées que pour les espèces qui ont une efficacité et une capturabilité proches de $50 \%$, de façon à vérifier si une absence apparente d'effet dû à la hauteur de piégeage pouvait être masquée par une interaction avec l'un des 2 autres facteurs. Ces résultats ne sont pas présentés dans le tableau mais sont utilisés directement dans le texte.

En utilisant ces différents critères, les espèces ont été séparées en 4 groupes (tableau VI) : les espèces pour lesquelles l'effet dû à la hauteur de piégeage est stable et important (effet 3), les espèces pour lesquelles l'effet dû à la hauteur de piégeage est presque stable (effet 2), les espèces pour lesquelles l'effet dû à la hauteur est faible ou présente de fortes interactions avec les facteurs de l'environnement (effet 1) et, enfin, les espèces qui ne présentent aucun effet dû à la hauteur de piégeage qui soit appréciable avec le protocole utilisé (effet 0 ). 
Espèces à effet hauteur stable et important (effet 3)

Ce sont les espèces pour lesquelles la différence de capturabilité entre les 2 hauteurs est hautement significative avec, dans plus de $80 \%$ des cas, des captures plus importantes soit à $0,7 \mathrm{~m}$, soit à $0,0 \mathrm{~m}$, et pour lesquelles la différence d'efficacité est également forte.

$T$ annulatus, $M$ castanicola, Thelaxes dryophila Schrank, Rhopalosiphum padi Linnaeus, $S$ avenae et $H$ lactucae composent ce groupe. Les 3 premiers sont presque exclusivement capturés à $0,7 \mathrm{~m}$. $R$ padi et $S$ avenae, 2 espèces de monocotylédones, sont également le plus souvent capturés en plus grand nombre à $0,7 \mathrm{~m}$, mais avec des différences d'efficacité moins importantes. $H$ lactucae, vivant sur dicotylédones, est à l'inverse presque toujours mieux capturée à 0,0 $m$ qu'à $0,7 \mathrm{~m}$.

Pour les pucerons des arbres, les rapports d'efficacité sont plus élevés $(>10)$ que pour les pucerons des plantes herbacées $(<5)$. La hau-

a

\section{Landerneau $0.7 \mathrm{~m}$}

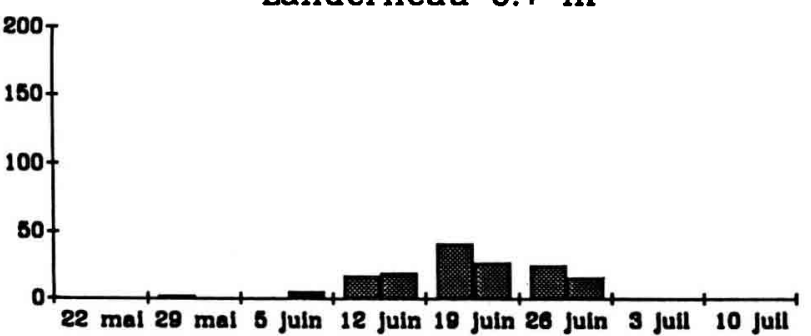

Pleyber $0.7 \mathrm{~m}$

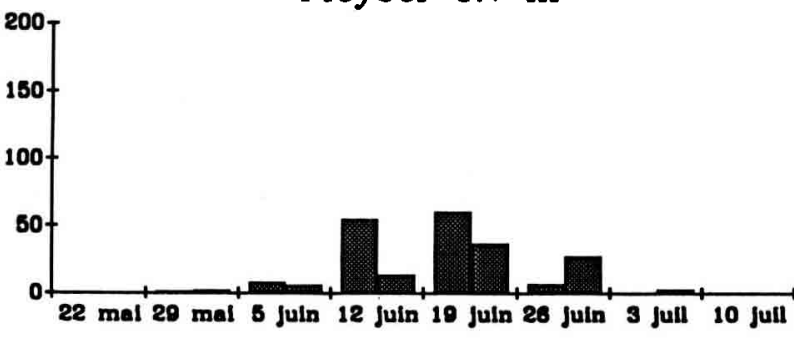

Chateaulin $0.7 \mathrm{~m}$

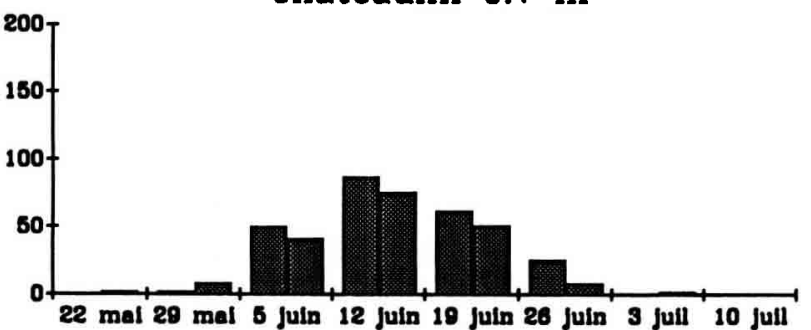

teur de piégeage est donc moins sélective pour ces dernières.

\section{Espèces à effet hauteur presque stable (effet 2)}

Ce sont les espèces pour lesquelles la différence de capturabilité est également significative, mais avec un nombre d'exceptions relativement important puisque dans 20 à $30 \%$ des cas on observe un effet opposé. Pour ces espèces, les différences d'efficacité sont variables. II s'agit de $B$ brassicae, de $S$ fragariae, de $M$ dirhodum, d'Aulacorthum solani Kaltenbach et d'Acyrthosiphon loti Theobalb.

Parmi ces espèces, $M$ dirhodum occupe une place particulière. Elle est, en effet, capturée en plus grand nombre à $0,7 \mathrm{~m}$ dans $80 \%$ des occasions, mais avec une différence d'efficacité globalement nulle, puisque 1485 individus ont été capturés au total à $0,7 \mathrm{~m}$ et que 1459 l'ont été à $0,0 \mathrm{~m}$. Cette absence apparente d'efficacité à $0,7 \mathrm{~m}$ est due à une capture exceptionnelle de 971 individus à Landerneau, en 1976, à $0,0 \mathrm{~m}$ pour seulement 172 individus à $0,7 \mathrm{~m}$. Cette in-

b

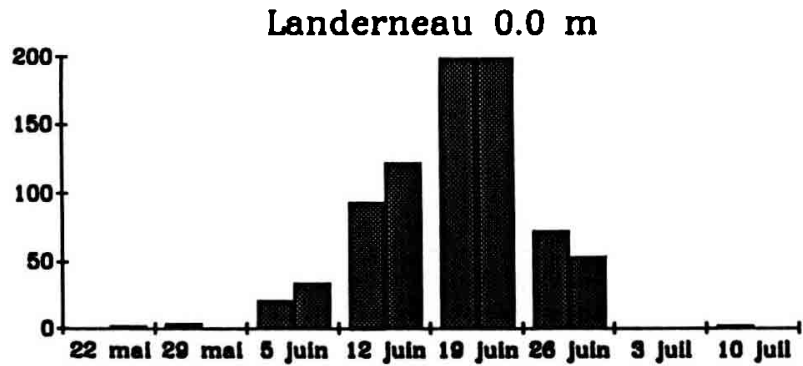

Pleyber $0.0 \mathrm{~m}$

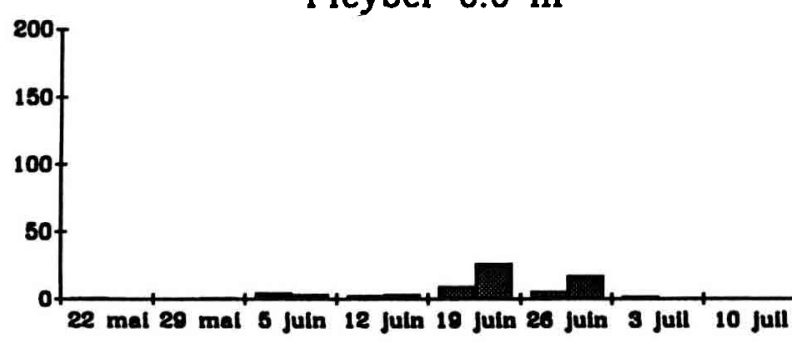

Chateaulin $0.0 \mathrm{~m}$

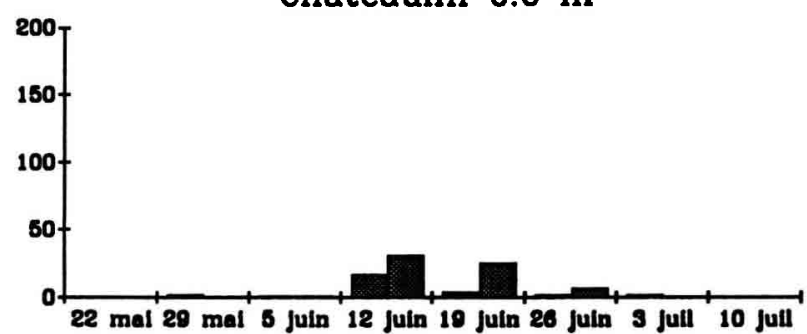

Fig 3. Captures hebdomadaires de Metopolophium dirhodum dans 3 sites en 1976. a. Résultats des 2 pièges exposés à $0,7 \mathrm{~m}$. b. Résultats des 2 pièges exposés à $0,0 \mathrm{~m}$. 
version d'effet s'observe pendant toute la période de piégeage de mai à juillet et dans chacune des 2 cuvettes exposées à $0,0 \mathrm{~m}$. De plus, l'évolution hebdomadaire des captures observée à Landerneau est comparable à celle des autres sites, comme Pleyber ou Châteaulin avec une seule période de vol présentant un maximum à la mi-juin (fig 3). Cette inversion d'effet n'est donc due ni à une capture exceptionnelle, voire accidentelle, ni à une périodicité particulière du vol ; elle est probablement due à un facteur d'hétérogénéité que le dispositif expérimental n'a pas permis de mettre en évidence.

Cette différence importante d'environ $900 \mathrm{M}$ dirhodum capturés en "surnombre" à $0,0 \mathrm{~m}$ à Landerneau en 1976 explique en bonne partie l'interaction entre la hauteur et le site décrite pour l'effectif total. Sans ce cas particulier, l'efficacité pour $M$ dirhodum serait de $73 \%$ à $0,7 \mathrm{~m}$.

Les cas de $S$ fragariae et de $B$ brassicae sont un peu similaires. Ces 2 espèces sont en moyenne capturées en plus grand nombre à 0,7 $\mathrm{m}$, sauf à Landerneau en 1975 et 1976, où les captures sont plus abondantes à $0,0 \mathrm{~m}$. Mais les inversions d'effet sont beaucoup moins importantes que pour $M$ dirhodum.

À l'inverse, $A$ solani et $A$ loti sont 2 espèces généralement mieux capturées à $0,0 \mathrm{~m}$ qu'à 0,7 $\mathrm{m}$. Ces différences d'efficacité et de capturabilité sont observées les années où ces espèces sont fortement capturées, ce qui a été le cas en 1974, 1975 et 1976 pour $A$ loti et en 1974 et 1975 pour $A$ solani. En revanche, ces relations peuvent s'inverser lorsque les captures sont faibles, $<20$ individus pour $A$ loti et $<10$ individus pour $A$ solani.

\section{Espèces à effet hauteur instable ou faible (effet 1)}

Ce sont les espèces caractérisées par une efficacité moyenne d'environ $60 \%$ des individus capturés soit à $0,7 \mathrm{~m}$, soit à $0,0 \mathrm{~m}$ mais avec une différence de capturabilité non significative. II s'agit de Metopolophium festucae Theobald, de Nasonovia ribisnigri Mosley, de Myzus certus Walker, d'Amphorophora rubi Kaltenbach et de Myzus persicae Sulzer.

$A$ pisum a également été rangée dans ce groupe bien qu'elle présente une différence de capturabilité significative, avec $69 \%$ d'occasions de captures plus importantes à $0,7 \mathrm{~m}$ qu'à $0,0 \mathrm{~m}$. Cependant, les $31 \%$ d'occasions où les captures sont moins importantes à $0,7 \mathrm{~m}$ ne se répartis- sent pas au hasard entre les différents sites, mais sont observées uniquement à Lesneven, Braspart et Landerneau. L'effet dû à la hauteur de piégeage interagit donc fortement avec le site et ne peut être considéré comme stable.

$M$ festucae présente une différence de capturabilité non significative. Cela est dû à un très grand nombre de captures nulles (15 occasions sur 35) obtenues en 1977 et 1978 . En revanche la capturabilité calculée sur les années 1974, 1975 et 1976 est significativement plus élevée à $0,7 \mathrm{~m}$ qu'à $0,0 \mathrm{~m}$.

$N$ ribisnigri, Myzus certus Walker, $M$ persicae et $A$ rubi sont, en moyenne, piégées plus efficacement à $0,0 \mathrm{~m}$ qu'à $0,7 \mathrm{~m}$. Mais ces espèces présentent des différences de capturabilité non significatives. Pour $N$ ribisnigri et $M$ certus, il n'y a pas d'interactions particulières entre la hauteur de piégeage et l'année ou le site. L'absence de différence significative de capturabilité est alors due à un grand nombre d'exceptions qui se répartissent au hasard parmi les 35 occasions. Pour $A$ rubi, l'absence de différence de capturabilité est due essentiellement à l'année 1976 où les captures ont été supérieures à $0,7 \mathrm{~m}$ pour 5 sites sur les 7. Pour $M$ persicae, cette absence de différence de capturabilité est due surtout à des captures faibles en 1977 et 1978.

\section{Espèces sans effet hauteur (effet 0 )}

Ce sont toutes des espèces pour lesquelles la différence de capturabilité n'est pas significative. Parmi ces espèces, certaines ne présentent également aucune différence d'efficacité. Ces espèces sont donc indifféremment capturées à 0,7 $\mathrm{m}$ ou à $0,0 \mathrm{~m}$ : il s'agit de Microlophium carnosum Buckton, d'Acyrthosiphon malvae Mosley, de Brachycaudus helichrysi Kaltenbach et de $C$ aegopodii.

Les cas de Macrosiphum euphorbiae Thomas et d'A fabae sont différents ; ces 2 espèces présentent une efficacité meilleure à $0,7 \mathrm{~m}$ pour $M$ euphorbiae et à $0,0 \mathrm{~m}$ pour $A$ fabae. Mais ces espèces sont également caractérisées par des interactions importantes entre l'effet dû à la hauteur de piégeage et les effets dus au site ou à l'année. Pour ces 2 espèces, aucune règle particulière n'a donc pu être décrite. Pour $A$ fabae, cependant, il existe une forte variabilité géographique : à Landerneau, Pleyber et Châteaulin, les captures sont sensiblement plus importantes à $0,0 \mathrm{~m}$ qu'à $0,7 \mathrm{~m}$, alors que l'inverse est observé à Rosporden, Lesneven et Braspart. 


\section{DISCUSSION ET CONCLUSION}

La comparaison des captures de pucerons ailés réalisées par pièges jaunes à 2 hauteurs, $0,0 \mathrm{~m}$ et $0,7 \mathrm{~m}$, fait apparaître une différence d'efficacité et de capturabilité des pièges. Cette différence s'applique aux caractéristiques globales des captures. On observe ainsi, en moyenne, un plus grand nombre d'individus et d'espèces à $0,7 \mathrm{~m}$ qu'à $0,0 \mathrm{~m}$. Les pièges à $0,7 \mathrm{~m}$ permettent donc, en première approximation, une meilleure estimation de la richesse et de la densité des populations aphidiennes en cours de déplacement. $\mathrm{Ne}$ disposer de pièges jaunes qu'à cette hauteur est donc suffisant pour un suivi régulier et plurilocal des pucerons.

Mais cette différence moyenne d'efficacité subit une forte variabilité géographique et surtout annuelle, car elle est très dépendante de la composition spécifique des peuplements échantillonnés. En effet, à l'exception de $C$ aegopodii et d' $A$ fabae, la plupart des espèces les plus abondamment capturées présentent une hétérogénéité verticale relativement stable. La variabilité géographique et annuelle de la répartition verticale des peuplements capturés s'explique donc essentiellement par la variabilité géographique et annuelle de l'importance relative des espèces les plus abondantes. Cette variabilité géographique et annuelle se superpose à l'influence de la variabilité saisonnière de la composition spécifique des peuplements sur l'efficacité des piégeages décrite par A'Brook (1973) et par Robert et al (1974). Tout protocole d'échantillonnage visant à suivre, au niveau des cultures, l'évolution de la densité aérienne des populations aphidiennes doit tenir compte de ces différentes échelles de variabilité. Dans le cas d'espèces pour lesquelles l'effet dû à la hauteur de piégeage est instable, la prise en compte des différentes échelles de variabilité peut conduire à devoir combiner plusieurs hauteurs de piégeage, voire plusieurs systèmes de piégeage.

Dans cette étude, l'appréciation de l'effet dû à la hauteur de piégeage est fondée pour chacune des 23 espèces sur les captures cumulées pendant toute la période d'exposition des pièges, de mai à juillet. La méthode ainsi utilisée peut donc éventuellement confondre 2 phases du vol des pucerons que sont la contamination en début de période, qui correspond à l'arrivée des pucerons dans les cultures, et la dissémination en fin de période qui comprend également la production d'ailés par la culture. Nos résultats permettent cependant de généraliser ceux de Robert et al (1974) pour 10 espèces sur les 19 qui sont communes aux 2 études.

Pour 7 autres espèces, nos résultats diffèrent de ceux de Robert et al (1974), mais les différences peuvent s'expliquer par la variabilité géographique et annuelle de la répartition verticale de ces espèces. Pour $S$ avenae, $S$ fragariae et $M$ dirhodum, ces auteurs concluent à une absence d'effet dû à la hauteur de piégeage. Or leur expérimentation a eu lieu lors d'années à faible abondance de ces espèces et, dans ce cas, nos travaux montrent effectivement que leur hétérogénéité verticale ne peut pas être démontrée. Pour $A$ rubi et $M$ persicae, ces auteurs, ainsi que A'Brook (1973) pour M persicae, ont des résultats comparables aux nôtres lors de certaines années à très fortes captures. Mais nos résultats montrent aussi que le nombre d'occasions de piégeage où il n'y a pas de différence significative entre les 2 hauteurs est trop important pour pouvoir conclure à une hétérogénéité stable de la répartition verticale de ces espèces.

Pour $A$ malvae et $A$ fabae, espèces pour lesquelles Robert et al (1974) concluent à une efficacité de piégeage supérieure à $0,0 \mathrm{~m}$, nous avons montré que la variabilité géographique ainsi que les interactions site-année ne permettent pas de généraliser des conclusions obtenues à partir d'un protocole trop restreint. II en est de même pour $M$ certus sans que nous ayons pu déceler des facteurs précis de variabilité, mais seulement un grand nombre d'occasions de piégeage sans différence de captures entre les 2 hauteurs.

Pour B brassicae, Robert et al (1974) concluent à une absence d'effet dû à la hauteur de piégeage. Nous concluons, au contraire, à une efficacité et à une capturabilité plus importantes à $0,7 \mathrm{~m}$. Leurs conclusions sont dues essentiellement aux captures effectuées à Rosporden en 1971 , où plus de 4000 individus de $B$ brassicae ont été capturés en se répartissant également entre les 2 hauteurs. Or, sur les 5 cinq années et les 7 sites de notre protocole, nous n'avons jamais capturé plus de 500 individus. L'expérimentation de Robert et al (1974) a peutêtre correspondu à une situation exceptionnelle pour la biologie et la dynamique de $B$ brassicae.

Par ailleurs, nos résultats sont identiques pour 7 des 11 espèces communes avec l'étude 
de Hullé et al (1992). II s'agit de $A$ fabae, $B$ brassicae, $B$ helichrysi, $C$ aegopodii, $H$ lactucae, $M$ persicae et $R$ padi. De même, nos résultats sont comparables pour 9 des 15 espèces communes avec le travail de Labonne et al (1989). II s'agit d'A fabae, $A$ solani, $B$ helichrysi, $B$ brassicae, $H$ lactucae, $M$ euphorbiae, $M$ castanicola, $N$ ribisnigri et $R$ padi. Ces 2 travaux, menés respectivement en Espagne et dans le Sud-Est de la France et donc dans 2 situations biogéographiques très différentes de la Bretagne, confèrent une certaine universalité aux relations décrites dans notre propre étude.

Au-delà de ces résultats, le présent travail permet d'alimenter une discussion plus large sur la provenance des pucerons capturés par pièges jaunes et de formuler quelques hypothèses.

Une différence d'efficacité et de capturabilité des espèces par pièges jaunes entre $0,0 \mathrm{~m}$ et $0,7 \mathrm{~m}$ correspond-elle à un gradient vertical de leur densité aérienne ? Les réponses à cette question reposent, d'une part, sur la connaissance du rayon d'attraction des pièges jaunes et, d'autre part, sur l'estimation de l'importance relative des composantes verticale et horizontale des vols. Les travaux d'A'Brook (1973) donnent a priori à la composante verticale du vol une grande importance puisqu'on réduit très sensiblement les captures en couvrant les pièges. Autrement dit, les pucerons semblent attirés par les pièges jaunes. Nous savons seulement, d'une part, qu'un puceron ne sera pas capturé s'il vole en dessous du piège et, d'autre part, que les fanes de pomme de terre ne couvrent jamais les pièges à $0,0 \mathrm{~m}$ et a fortiori à $0,7 \mathrm{~m}$.

Dans le cas présent, où la végétation environnante ne concurrence pas les pièges de manière importante, les différences de capturabilité que nous observons ne peuvent pas s'expliquer si les pucerons sont attirés de haut vers le jaune lors d'un vol de migration qui se situerait audessus de la couche limite de vol actif. II nous semble, au contraire, que les captures par pièges jaunes correspondent à un vol actif, de proximité par rapport au support végétal plutôt qu'à un "atterrissage" des pucerons à partir d'un vol forcé dû à un entraînement aérien. Cette hypothèse n'est pas en contradiction avec les résultats d'A'Brook (1973) qui montrent, pour plusieurs espèces, un gradient vertical régulier de la densité des captures par pièges jaunes entre $0,0 \mathrm{~m}, 0,46 \mathrm{~m}$ et $0,91 \mathrm{~m}$. Si l'influence du couvert végétal n'intervient pas, un tel gradient ne peut alors s'expliquer que par une composante horizontale importante dans la direction de vol de ces espèces. Autrement dit, chaque espèce aurait une hauteur de vol actif préférentielle, ce qui traduirait les différences de capturabilité que nous observons.

L'hypothèse de Taylor (1974) sur l'existence d'une couche limite en deçà de laquelle les pucerons ont un vol volontaire et dirigé offre un cadre général qui permet d'accueillir l'ensemble de ces résultats et les hypothèses qu'ils suscitent. En effet, si une telle couche limite existe pour les pucerons, elle doit se traduire par des différences de densité et de capturabilité dans les premiers décimètres au-dessus du sol.

\section{RÉFÉRENCES}

A'Brook J (1973) Observations on different methods of aphid trapping. Ann App/ Biol 74, 263-277

Hullé M, Seco MV, Nieto JM (1992) Comparación de la estructura de las poblaciones de áfidos (Hom Aphididae) alados capturados en León por diferentes tipos de trampas. Orsis 7, 65-77

Johnson CG, Taylor LR (1955) The development of large suction traps for airbone insects. Ann Appl Biol 43, 51-62

Labonne G, Fauvel G, Leclant F, Quiot JB (1983) Intérêt des pièges à fils dans l'étude des populations de pucerons ailés. agronomie 3, 315-326

Labonne G, Lauriaut F, Quiot JB (1989) Comparaison de trois types de pièges pour l'échantillonnage des populations des pucerons ailés. agronomie 9, 547557

Lebart L, Morineau A, Fénelon JP (1979) Traitement des données statistiques, méthodes et programmes. Dunod, Paris

Lebart L, Morineau A, Lambert T (1988) SPAD.N : système portable pour l'analyse des données, version 1.2, Manuel de référence. CISIA, Sèvres

Moericke V (1951) Eine Farbfalle zur Kontrolle des Fluges von Blattläusen, insbesondere des Pfirsichblattlaus, Myzodes persicae (Sulz). Nachrichtenbl Dtsch Pflanzeschutzdienstes Dienot (Braunschw) 3, 23-24

Robert Y, Rouzé-Jouan J (1976) Activité saisonnière de vol des pucerons dans l'Ouest de la France. Résultats de neuf années de piégeage (1967-1975). Ann Soc Entomol Fr (NS) 12(4), 671-690

Robert Y, Rabasse JM, Rouzé-Jouan J (1974) Sur l'utilisation des pièges jaunes pour la capture de pucerons en culture de pomme de terre. I. Influence de la hauteur de piégeage. Ann Zool Ecol Anim 6 (3), 349-372 
Robert Y, Dedryver CA, Pierre JS (1988) Sampling Techniques. In: Aphids, their biology, natural enemies and control (AK Minks, P Harrewijn, eds). Elsevier, Amsterdam, vol B, 1-20

Taylor LR (1974) Insect migration, flight periodicity and the boundary layer. J Anim Ecol 43, 225-238
Taylor LR, Palmer JMP (1972) Aerial Sampling. In: Aphid technology (HF Van Emdem, ed) Academic Press, Londres, 189-234

Tranchefort J, Beaux MF, Gouet H, Gouet JP, Philippeau G, Verneau M (1987) STATITCF. Manuel d'utilisation. ITCF, Paris 